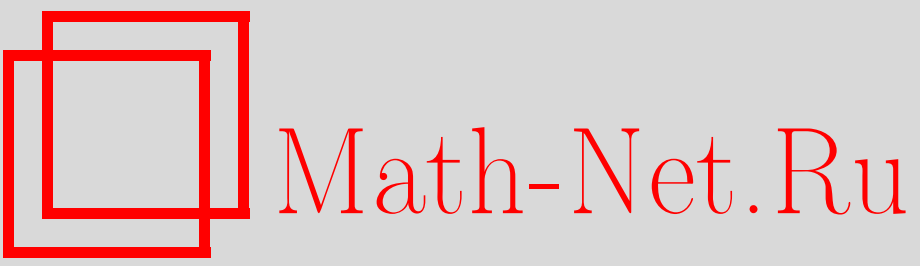

Е. Н. Гетманова, С. В. Корнев, О некоторых применениях теории случайной степени совпадения в периодической задаче для функциональнодифференциальных включений, Итоги науки и техн. Сер. Соврем. мат. и ее прил. Темат. обз., 2020, том 186, 21-31

DOI: https://doi.org/10.36535/0233-6723-2020-186-21-31

Использование Общероссийского математического портала Math-Net.Ru подразумевает, что вы прочитали и согласны с пользовательским соглашением

http://www. mathnet.ru/rus/agreement

Параметры загрузки:

IP : 54.162 .127 .20

26 апреля 2023 г., 09:55:11 


\title{
О НЕКОТОРЫХ ПРИМЕНЕНИЯХ ТЕОРИИ СЛУЧАЙНОЙ СТЕПЕНИ СОВПАДЕНИЯ В ПЕРИОДИЧЕСКОЙ ЗАДАЧЕ ДЛЯ ФУНКЦИОНАЛЬНО-ДИФФЕРЕНЦИАЛЬНЫХ ВКЛЮЧЕНИЙ
}

\author{
(c) 2020 г. $\quad$ E. H. ГЕTMAHOBA, C. B. KOPHEB
}

\begin{abstract}
АннотАция. Метод случайных интегральных направляющих функций, разработанный на основе теории случайной топологической степени совпадения, применен к исследованию периодической задачи для случайных функционально-дифференциальных включений в конечномерных пространствах.
\end{abstract}

Ключевые слова: случайная интегральная направляющая функция, случайный потенциал, случайный мультиоператор, случайная степень совпадения, случайный топологический индекс.

\section{SOME APPLICATIONS OF THE THEORY OF RANDOM DEGREE OF COINCIDENCE IN PERIODIC PROBLEMS FOR FUNCTIONAL DIFFERENTIAL INCLUSIONS}

\author{
(c) 2020 E. N. GETMANOVA, S. V. KORNEV
}

\begin{abstract}
The method of random integral direction functions developed on the basis of the theory of a random topological degree of coincidence is applied to the study of the periodic problem for random functional differential inclusions in finite-dimensional spaces.

Keywords and phrases: random integral directing function, random potential, random multioperator, random degree of coincidence, random topological index.
\end{abstract}

AMS Subject Classification: 34F05, 34A60, 34C25

1. Введение. Одним из наиболее эффективных и геометрически наглядных способов решения задачи о существовании периодических колебаний дифференциальных уравнений является метод направляющих функций, общие принципы которого сформулировали еще в середине ХХ века М. А. Красносельский и А. И. Перов (см. [14,15]). В конце XX - начале XXI веков в связи с открывшимися новыми возможностями приложений к актуальным задачам математики, механики и теории управления, возникла необходимость в существенном расширении классов рассматриваемых направляющих функций (см., например, $[2,8,10,12,18,21,22,26,26,34,37])$.

Одними из активно изучаемых в последние годы динамических систем являются системы, описываемые случайными дифференциальными включениями (см., например, $[2,3,8-10,12,14,15,18$, $18,19,21,23,26,27,30,31,34])$. В настоящей работе для исследования задачи о существовании периодических колебаний случайных функционально-дифференциальных включений предлагается применить метод случайных интегральных направляющих функций. 
2. Предварительные сведения. Напомним основные понятия теории многозначных отображений (см., например, $[1,2,17,19,20,23,25])$ и теории степени совпадения (см., например, $[9,31,35,36,38])$.

Пусть $\left(X, d_{X}\right)$ и $\left(Y, d_{Y}\right)$ являются метрическими пространствами и $I=[0, T]$. Символами $P(Y)$, $C(Y), K(Y)$ обозначаются совокупности всех, соответственно, непустых, замкнутых или компактных подмножеств пространства $Y$. Если $Y$ - нормированное пространство, то символами $C v(Y), K v(Y)$ обозначаются совокупности всех непустых выпуклых, соответственно, замкнутых или компактных подмножеств пространства $Y$.

Определение 1. Мультиотображение $F: X \rightarrow P(Y)$ называется полунепрерывным сверху (nн. св.) в точке $x_{0} \in X$, если для любого $\varepsilon>0$ найдется $\delta>0$ такое, что из того, что $d_{X}\left(x_{0}, x\right)<\delta$ следует, что $F(x) \subset U_{\varepsilon}\left(F\left(x_{0}\right)\right)$, где символ $U_{\varepsilon}$ обозначает $\varepsilon$-окрестность множества.

Мультиотображение $F: X \rightarrow P(Y)$ называется полунепрерывным сверху (пн. св.), если оно пн. св. в каждой точке $x \in X$.

Определение 2. Множество $A \subset X$ называется стягиваемым, если существует непрерывное отображение (гомотопия) $h: A \times[0,1] \rightarrow A$ такое, что $h(x, 0)=x, h(x, 1)=x_{0}$ для всех $x \in A$.

Определение 3. Множество $A \subset X$ называется $R_{\delta}$-множеством, если существует убывающая последовательность $\left\{A_{n}\right\}$ компактных стягиваемых множеств таких, что

$$
A=\bigcap\left\{A_{n}: n=1,2, \ldots\right\} .
$$

Определение 4. Полунепрерывное сверху мультиотображение $G: X \rightarrow K(Y)$ называется $\mathcal{J}$ отображением, или $G \in \mathcal{J}(X, Y)$, если для любого $x \in X$ множество $G(x)$ является $R_{\delta}$-множеством.

Определение 5. ${ }^{c} J$-мультиотображением называется конечная композиция полунепрерывных сверху мультиотображений с $R_{\delta}$-значениями.

Определение 6. Мультиотображение $F: X \rightarrow P(Y)$ называется компактным, если область значений $F(X)$ относительно компактна в $Y$, т. е. $\overline{F(X)}$ компактно в $Y$.

В дальнейшем пн. св. и компактное мультиотображение $F: X \rightarrow P(Y)$ будем называть просто компактным.

Мультиотображение будем называть мультифункиией, если оно определено на подмножестве числовой прямой.

Пусть $I$ - замкнутое подмножество $\mathbb{R}$, снабженное мерой Лебега.

Определение 7. Мультифункция $F: I \rightarrow K(Y)$ называется измеримой, если для любого открытого подмножества $W \subset Y$ его прообраз

$$
F^{-1}(W)=\{t \in I: F(t) \subset W\}-
$$

измеримое подмножество $I$.

Пусть $E_{1}, E_{2}$ - банаховы пространства, $l: \operatorname{dom} l \subseteq E_{1} \rightarrow E_{2}$-линейный (необязательно непрерывный) оператор.

Напомним о следующих известных фактах (см., например, [31]).

Теорема 1. Пусть $p: E_{1} \rightarrow E_{1}$-линейный оператор проектирования такой, что $\operatorname{Im} p=$ Ker $l$. Тогда:

1. оператор $l_{p}: \operatorname{dom} l \cap \operatorname{Ker} p \rightarrow \operatorname{Im} l$, заданный как

$$
l_{p}(x)=l(x) \text { для всех } x \in \operatorname{dom} l \cap \operatorname{Ker} p
$$

является линейным изоморбизмом; 
2. оператор $k_{p}: \operatorname{Im} l \rightarrow \operatorname{dom} l \cap \operatorname{Ker} p$, заданный как

$$
k_{p}=l_{p}^{-1},
$$

удовлетворяет соотношению

$$
k_{p} \circ l(x)=x-p(x) \text { для всех } x \in \operatorname{dom} l .
$$

Определение 8. Линейный оператор $l: \operatorname{dom} l \subseteq E_{1} \rightarrow E_{2}$ называется линейным фредгольмовым оператором нулевого индекса, если $\operatorname{Ker} l$ и $\operatorname{Coker} l=E_{2} / \operatorname{Im} l$ имеют конечную размерность и

$$
\operatorname{dim} \operatorname{Ker} l=\operatorname{dim} \text { Coker } l .
$$

Теорема 2. Пусть $l: \operatorname{dom} l \rightarrow E_{2}-$ линейный фредгольмов оператор нулевого индекса такой, что $\operatorname{Im} l \subset E_{2}-$ замкнутое множество. Тогда справедливы следующие утверждения:

(1) существуют линейные непрерывные операторы проектирования $p: E_{1} \rightarrow E_{2}, q: E_{2} \rightarrow E_{2}$ такие, что $\operatorname{Im} p=\operatorname{Ker} l u \operatorname{Im} l=\operatorname{Ker} q$;

(2) канонический оператор проектирования $\pi: E_{2} \rightarrow E_{2} / \operatorname{Im} l$, заданный как

$$
\pi(y)=y+\operatorname{Im} l
$$

является непрерывным линейным оператором;

(3) существует непрерывный линейный изоморфизм $\phi:$ Coker $l \rightarrow \operatorname{Ker} l$;

(4) уравнение $l(x)=y, y \in E_{2}$, является эквивалентным уравнению $(i-p) x=\left(\phi \circ \pi+k_{p, q}\right)(y)$, где $i$-тождественный оператор в $E_{1}$, а оператор $k_{p, q}: E_{2} \rightarrow E_{1}$ задан соотношением

$$
k_{p, q}(y)=k_{p}(y-q(y)) .
$$

Пусть $U$ - открытое ограниченное подмножество $E_{1}$.

Определение 9. Мультиотображение $F \in^{c} J\left(\bar{U}, E_{2}\right)$ называется $l$-компактным, если композиция

$$
\left(\phi \circ \pi+k_{p, q}\right) \circ F: \bar{U} \rightarrow K\left(E_{1}\right)
$$

является компактным мультиотображением.

Замечание 1 (см., например, [31]). Приведенное выше определение $l$-компактного мультиотображения не зависит от выбора линейных операторов проектирования $p: E_{1} \rightarrow E_{2}$ и $q: E_{1} \rightarrow$ $E_{2}$, а также изоморфизма $\phi: \operatorname{Coker} l \rightarrow \operatorname{Ker} l$.

Приведем теперь некоторые сведения из теории случайных многозначных отображений и случайной топологической степени (см., например, $[3,18,39])$.

Пусть $(\Omega, \Sigma, \mu)$ - полное вероятностное пространство.

Определение 10. Мультиотображение $\mathcal{F}: \Omega \times X \rightarrow C(Y)$ называется случайным мультиоnератором, если он измерим относительно $\Sigma \otimes \mathbb{B}(X)$, где $\Sigma \otimes \mathbb{B}(X)$ - наименьшая $\sigma$-алгебра на $\Omega \times X$, включающая все множества $A \times B$, где $A \in \Sigma, B \in \mathbb{B}(X)$ и $\mathbb{B}(X)$ обозначает борелевскую $\sigma$-алгебру на $X$. Если, кроме того, $\mathcal{F}(\omega, \cdot): X \rightarrow C(Y)$ полунепрерывно сверху для всех $\omega \in \Omega$, то $\mathcal{F}$ называется случайным и-мультиоператором.

Определение 11. Пусть $A \subset Y$ - замкнутое подмножество и $\mathcal{F}: \Omega \times A \rightarrow P(Y)$ - случайный мультиоператор. Случайной неподвижной точкой $\xi$ мультиоператора $\mathcal{F}$ называется измеримая функция $\xi: \Omega \rightarrow A$ такая, что

$$
\xi(\omega) \in \mathcal{F}(\omega, \xi(\omega)) \text { для всех } \omega \in \Omega .
$$

Теорема 3 (см. [18]). Пусть $Y$ - сепарабельное банахово пространство, $\mathcal{F}: \Omega \times Y \rightarrow C(Y)-$ случайный и-мультиоператор. Если для каждого $\omega \in \Omega$ множество

$$
F_{i x} \mathcal{F}_{\omega}:=\{x \in Y: x \in \mathcal{F}(\omega, x)\}
$$

неподвижных точек мультиоператора $\mathcal{F}_{\omega}=\mathcal{F}(\omega, \cdot)$ непусто и замкнуто, то $\mathcal{F}$ имеет случайную неподвижную точку. 
Определение 12. Мультиоператор $\mathcal{F}: \Omega \times X \rightarrow K(Y)$ называется случайным компактным $u$-мультиоператором, если он является случайным $u$-мультиоператором и для каждого $\omega \in \Omega$ мультиоператор $\mathcal{F}(\omega, \cdot): X \rightarrow K(Y)$ является компактным.

Пусть $Y$ - сепарабельное банахово пространство, $U \subset Y$ - открытое ограниченное подмножество и $\mathcal{F}: \Omega \times \bar{U} \rightarrow K v(Y)$ - случайный компактный $u$-мультиоператор такой, что $x \notin \mathcal{F}(\omega, x)$ для всех $x \in \partial U$ и для всех $\omega \in \Omega$, где $\partial U$ обозначает границу множества $U$. Тогда для каждого $\omega \in \Omega$ топологическая степень соответствующего многозначного векторного поля $\operatorname{deg}(i-\mathcal{F}(\omega, \cdot), \bar{U})$ корректно определена (см., например, $[2,19,20,23])$. Случайная топологическая степень многозначного векторного поля $i-\mathcal{F}$ на $\bar{U}$ определяется следующим образом (см. [18]):

$$
D(i-\mathcal{F}, \bar{U}):=\{\operatorname{deg}(i-\mathcal{F}(\omega, \cdot), \bar{U}) \mid \omega \in \Omega\} .
$$

Из того, что топологическая степень $\operatorname{deg}(i-\mathcal{F}(\omega, \cdot), \bar{U}) \neq 0$ для всех $\omega \in \Omega$ следует, что случайная топологическая степень $D(i-\mathcal{F}, \bar{U}) \neq 0$.

Теорема 4 (см. [18]). Если случайная топологическал степень $D(i-\mathcal{F}, \bar{U}) \neq 0$, то мультиоператор $\mathcal{F}$ имеет случайную неподвижную точку в $U, m$. е. существует измеримая функиия $\xi: \Omega \rightarrow U$ такал, что $\xi(\omega) \in \mathcal{F}(\omega, \xi(\omega))$ для всех $\omega \in \Omega$.

Пусть $X, Y$ - сепарабельные банаховы пространства, $U \subset Y$-открытое ограниченное подмножество, $l: \operatorname{dom} l \subseteq X \rightarrow Y$-линейный фредгольмов оператор нулевого индекса и случайный мультиоператор $\mathcal{F}: \bar{\Omega} \times \bar{U} \rightarrow C v(Y)$ удовлетворяет следующим условиям:

(1) для каждого $\omega \in \Omega$ мультиотображение $\mathcal{F}(\omega, \cdot)$ является $l$-компактным мультиотображением;

(2) для каждого $\omega \in \Omega$ мультиотображение $\mathcal{F}(\omega, \cdot)$ является ${ }^{c} J$-мультиотображением;

(3) для всех $x \in \partial U \cap \operatorname{dom} l$ и $\omega \in \Omega$

$$
l x \notin \mathcal{F}(\omega, x) .
$$

Тогда для каждого $\omega \in \Omega$ топологическая степень совпадения пары $(l, \mathcal{F}(\omega, \cdot))$ определяется как (см., например, [35,38])

$$
\operatorname{deg}(l, \mathcal{F}(\omega, \cdot), \bar{U}):=\operatorname{deg}(\Phi(\omega, \cdot), \bar{U}),
$$

где

$$
\Phi(\omega, x)=p(x)+\left(\phi \circ \pi+k_{p, q}\right) \circ \mathcal{F}(\omega, x) .
$$

Случайная топологическая степень совпадения пары $(l, \mathcal{F})$ определяется следующим образом (см., например, [39]):

$$
\operatorname{Deg}(l, \mathcal{F}, \bar{U}):=\{\operatorname{deg}(l, \mathcal{F}(\omega, \cdot), \bar{U}) \mid \omega \in \Omega\}
$$

Говорят, что случайная топологическая степень совпадения $\operatorname{Deg}(l, \mathcal{F}, \bar{U})$ пары $(l, \mathcal{F})$ отлична от нуля, если топологическая степень совпадения $\operatorname{deg}(l, \mathcal{F}(\omega, \cdot), \bar{U}) \neq 0$ для всех $\omega \in \Omega$.

Определенная таким образом случайная топологическая степень совпадения обладает всеми основными свойствами степени совпадения. В частности, справедлив следующий общий принцип (см., например, [39]).

Теорема 5. Если случайная топологическая степень совпадения $\operatorname{Deg}(l, \mathcal{F}, \bar{U}) \neq 0$, то существует случайная точка совпадения пары для данной пары $(l, \mathcal{F})$, m. е. измеримая функиия $\xi: \Omega \rightarrow U$ такал, что $l \xi(\omega) \in \mathcal{F}(\omega, \xi(\omega))$ для всех $\omega \in \Omega$.

Примером реализации приведенного общего принципа является следующее утверждение (см., например, [39]).

Теорема 6. Пусть случайное мультиотображение $\mathcal{F}: \Omega \times \bar{U} \rightarrow K\left(E_{2}\right)$ является l-компактным ${ }^{c} J$-мультиотображсением при каждом $\omega \in \Omega$ и выполнены следующие условия:

(1) $l x \notin \lambda \mathcal{F}(\omega, x)$ для каждого $\omega \in \Omega$, для всех $0<\lambda<1, x \in \operatorname{dom} l \cap \partial U$;

(2) $0 \notin \pi \mathcal{F}(\omega, x)$ для кажсдого $\omega \in \Omega$, для всех $x \in \operatorname{Ker} l \cap \partial U$;

(3) $\operatorname{deg}_{\operatorname{Ker} l}\left(\left.\phi \pi \mathcal{F}(\omega, \cdot)\right|_{\bar{U}_{\text {Ker }} l} \bar{U}_{\text {Ker } l}\right) \neq 0$ для каждого $\omega \in \Omega$, где символ $\operatorname{deg}_{\text {Ker } l}$ обозначает топологическую степень ${ }^{c} J$-мультиотображения $\phi \pi \mathcal{F}(\omega, \cdot)$, вычисляемую в конечномерном пространстве $\operatorname{Ker} l$, a $\bar{U}_{\operatorname{Ker} l}=\bar{U} \cap \operatorname{Ker} l$. 
Тогда случайная топологическая степень совпадения $\operatorname{Deg}(l, \mathcal{F}, U) \neq 0, u$, следовательно, существует случайная точка совпадения пары $(l, \mathcal{F})$.

3. Основной результат. Для $\tau>0$ обозначим символом $\mathcal{C}$ пространство $C\left([-\tau, 0] ; \mathbb{R}^{n}\right)$ непрерывных функций $x:[-\tau, 0] \rightarrow \mathbb{R}^{n}$ с нормой $\|x\|=\sup _{t \in[-\tau, 0]}\|x(t)\|$, и пусть $I=[0, T], T>0$. Для функции $x(\cdot) \in C\left([-\tau, T] ; \mathbb{R}^{n}\right)$ символом $x_{t} \in \mathcal{C}$ обозначается функция, заданная как $x_{t}(\theta)=x(t+\theta), \theta \in[-\tau, 0]$.

Будем рассматривать периодическую задачу для случайного функционально-дифференциального включения следующего вида:

$$
\begin{array}{r}
x^{\prime}(\omega, t) \in \mathcal{F}\left(\omega, t, x_{t}\right), \\
x(\omega, 0)=x(\omega, T),
\end{array}
$$

для всех $\omega \in \Omega$, где мультиотображение $\mathcal{F}: \Omega \times \mathbb{R} \times \mathcal{C} \multimap \mathbb{R}^{n}$ удовлетворяет следующим условиям:

$\left(\mathcal{F}_{t}\right)$ мультифункция $\mathcal{F}$ по второму аргументу $T$-периодична:

$$
\mathcal{F}(\omega, t, \phi)=F(\omega, t+T, \phi) \text { для всех } \omega \in \Omega, t \in \mathbb{R}, \phi \in \mathcal{C}
$$

(очевидно, это условие позволяет рассматривать мультиотображение $F$ заданным на $I \times \mathcal{C}$ ); $(\mathcal{F} 1) \mathcal{F}: \Omega \times I \times \mathcal{C} \rightarrow K v\left(\mathbb{R}^{n}\right)$ является случайным $u$-мультиоператором;

$(\mathcal{F} 2)$ существует отображение $c: \Omega \times I \rightarrow \mathbb{R}$ такое, что $c(\omega, \cdot)$ - локально интегрируемо на $\mathbb{R}$ для каждого $\omega \in \Omega, c(\cdot, t)$ - измеримо п. в. $t \in I$, и

$$
\|\mathcal{F}(\omega, t, \phi)\|:=\sup \{|z|: z \in \mathcal{F}(\omega, t, \phi)\} \leqslant c(\omega, t)(1+|\phi|) .
$$

Под случайным решением задачи (1), (2) понимается функция $\xi: \Omega \times I \rightarrow \mathbb{R}^{n}$ такая, что

(i) оператор $\omega \in \Omega \rightarrow \xi(\omega, \cdot) \in C\left([-\tau, T] ; \mathbb{R}^{n}\right)$ измерим;

(ii) для каждого $\omega \in \Omega$ абсолютно непрерывная функция $\xi(\omega, \cdot) \in C\left([-\tau, T] ; \mathbb{R}^{n}\right)$ удовлетворяет $(1),(2)$.

Из условий $(\mathcal{F} 1),(\mathcal{F} 2)$ следует, что мультиоператор суперпозиции

$$
\begin{gathered}
\mathcal{P}_{\mathcal{F}}: \Omega \times C\left([-\tau, T], \mathbb{R}^{n}\right) \multimap P\left(L^{2}\left(I, \mathbb{R}^{n}\right)\right), \\
\mathcal{P}_{\mathcal{F}}\left(\omega, x_{t}\right)=\left\{f \in L^{2}\left(I, \mathbb{R}^{n}\right): f(s) \in \mathcal{F}\left(\omega, s, x_{s}\right)\right\} \text { п. в. } s \in I
\end{gathered}
$$

корректно определен. Кроме того, для каждого $\omega \in \Omega$ мультиоператор $\mathcal{P}_{\mathcal{F}}(\omega, \cdot)$ замкнут (см., например, [2]).

Для изучении задачи (1), (2) мы будем использовать теорию случайной топологической степени совпадения пары отображений, изложенную в предыдущем параграфе.

Обозначим $C_{T}$ пространство непрерывных $T$-периодических функций $x: \mathbb{R} \rightarrow \mathbb{R}^{n}$ с нормой $\|x\|_{C}=\sup \|x(t)\|$. Через $\|x\|_{2}$ обозначим норму функции $x$ в пространстве $L^{2}$,

$$
\|x\|_{2}=\left(\int_{0}^{T}\|x(s)\|^{2} d s\right)^{\frac{1}{2}} .
$$

Определение 13 (см. [18]). Отображение $V: \Omega \times \mathbb{R}^{n} \rightarrow \mathbb{R}$ называется случайным потенииалом, если выполнены следующие условия:

(i) $V(\cdot, x): \Omega \rightarrow \mathbb{R}$ является измеримым для каждого $x \in \mathbb{R}^{n}$;

(ii) $V(\omega, \cdot): \mathbb{R}^{n} \rightarrow \mathbb{R}$ является $C^{1}$-отображением для каждого $\omega \in \Omega$.

Определение 14 (см. [18]). Случайный потенциал $V$ называется случайным невырожденным потенииалом, если найдется $R_{0}>0$ такое, что

$$
\nabla V(\omega, z)=\left(\frac{\partial V(\omega, z)}{\partial z_{1}}, \ldots, \frac{\partial V(\omega, z)}{\partial z_{n}}\right) \neq 0
$$

для всех $(\omega, z) \in \Omega \times \mathbb{R}^{n}:|z| \geqslant R_{0}$. 
Из определения 14 следует, что для фиксированного $\omega \in \Omega$ топологическая степень

$$
\operatorname{deg}\left(\nabla V(\omega, \cdot), B_{\mathbb{R}^{n}}(0, R)\right)
$$

корректно определена для всех $R \geqslant R_{0}$ и совпадает с $\operatorname{deg}\left(\nabla V(\omega, \cdot), B_{\mathbb{R}^{n}}\left(0, R_{0}\right)\right)$.

Под случайным топологическим индексом ind $V$ случайного невырожденного потенциала $V$ понимается случайная топологическая степень $\operatorname{Deg}\left(\nabla V, B_{\mathbb{R}^{n}}\left(0, R_{0}\right)\right)$.

Определение 15. Случайный потенциал $V: \Omega \times \mathbb{R}^{n} \rightarrow \mathbb{R}$ называется случайной строгой интегральной направляющей функиией для включения (1), если найдется $N>0$ такое, что для всех $\omega \in \Omega$ из $x \in C\left(I, \mathbb{R}^{n}\right)$ с $\|x\|_{2} \geqslant N$ следует, что

$$
\int_{0}^{T}\langle\nabla V(\omega, x(s)), f(s)\rangle d s>0 \text { для всех } f \in \mathcal{P}_{\mathcal{F}}\left(\omega, x_{s}\right) .
$$

Нетрудно убедиться в справедливости следующего утверждения.

Лемма 1. Если $V: \Omega \times \mathbb{R}^{n} \rightarrow \mathbb{R}$ является случайной строгой интегральной направляющей функиией для включения (1), то она является случайным невырожденным потенииалом, и, следовательно, существует ее случайный топологический индекс ind $V$.

Теорема 7. Пусть выполнены условия $\left(\mathcal{F}_{t}\right),(\mathcal{F} 1),(\mathcal{F} 2)$. Если $V: \Omega \times \mathbb{R}^{n} \rightarrow \mathbb{R}$ является строгой случайной интегральной направляющей функиией для включения (1) такой, что ind $V \neq 0$, то задача (1), (2) имеет случайное решение.

Замечание 2. Отметим, что условия теоремы выполнены, если, например, для каждого $\omega \in \Omega$ функция $V$ четна, или $\lim _{\|x\| \rightarrow+\infty} V(\omega, x)= \pm \infty$.

Доказательство. Для доказательства воспользуемся Теоремой 6. Рассмотрим следующие операторы:

$$
\begin{gathered}
l: \operatorname{dom} l:=\left\{x \in C_{T}: x-\text { абсолютно непрерывна }\right\} \subset C_{T} \rightarrow L_{T}^{1}, \\
l(x)=x^{\prime}
\end{gathered}
$$

и мультиоператор суперпозиции $\mathcal{G}=\mathcal{P}_{\mathcal{F}}: \Omega \times C_{T} \multimap P\left(L_{T}^{1}\right)$.

Легко видеть, что $l$ - линейный фредгольмов оператор нулевого индекса, $\operatorname{Ker} l=\mathbb{R}^{n}$. Проекция $\pi: L_{T}^{1} \rightarrow \mathbb{R}^{n}$ может быть задана формулой

$$
\pi f=\frac{1}{T} \int_{0}^{T} f(s) d s .
$$

Отметим, что для произвольного фиксированного $\omega \in \Omega$ решение $x_{\omega} \in \operatorname{dom} l$ включения $l(x) \in$ $\lambda \mathcal{G}\left(\omega, x_{t}\right), \lambda \in(0,1)$, удовлетворяет задаче

$$
\begin{gathered}
x_{\omega}^{\prime}(t)=\lambda f_{\omega}(t) \text { п. в. } t \in[0, T], \\
x_{\omega}(0)=x_{\omega}(T),
\end{gathered}
$$

где $f_{\omega} \in \mathcal{P}_{\mathcal{F}}\left(\omega, x_{t}\right)$.

Тогда

$$
\begin{aligned}
\int_{0}^{T}\left\langle\nabla V\left(\omega, x_{\omega}(s)\right), f_{\omega}(s)\right\rangle d s=\frac{1}{\lambda} \int_{0}^{T}\left\langle\nabla V\left(\omega, x_{\omega}(s)\right), x_{\omega}^{\prime}(s)\right\rangle d s & = \\
=\frac{1}{\lambda} \int_{0}^{T} V^{\prime}\left(\omega, x_{\omega}(s)\right) d s & =\frac{1}{\lambda}\left(V\left(\omega, x_{\omega}(T)\right)-V\left(\omega, x_{\omega}(0)\right)\right)=0
\end{aligned}
$$


откуда следует, что

$$
\left\|x_{\omega}\right\|_{2}<N
$$

С другой стороны, из условия $\left(\mathcal{F}_{2}\right)$ вытекает, что $\left\|x^{\prime}\right\|_{2}<M$, где $M>0$. Но тогда найдется и $M^{\prime}>0$ такое, что

$$
\|x\|_{C}<M^{\prime}
$$

В качестве $U$ возьмем шар $B_{r} \subset C_{T}$ радиуса $r=\max \left\{N, M^{\prime}, N T^{-1 / 2}\right\}$. Тогда имеем для каждого фиксированного $\omega \in \Omega$

$$
l(x) \notin \lambda \mathcal{G}\left(\omega, x_{t}\right)
$$

для всех $x \in \partial U, \lambda \in(0,1)$.

Пусть теперь $u \in \partial U \cap \operatorname{Ker} l$ произвольно. Поскольку $\|u\| \geqslant N T^{-1 / 2}$, из определения случайной строгой интегральной направляющей функции получаем, что

$$
\int_{0}^{T}\left\langle\nabla V(\omega, u), f_{\omega}(s)\right\rangle d s>0
$$

для любого измеримого сечения $f_{\omega}(s) \in \mathcal{P}_{\mathcal{F}}(\omega, u)$, для всех $\omega \in \Omega$. Но для каждого $\omega \in \Omega$

$$
\int_{0}^{T}\left\langle\nabla V(\omega, u), f_{\omega}(s)\right\rangle d s=\left\langle\nabla V(\omega, u), \int_{0}^{T} f_{\omega}(s) d s\right\rangle=T\left\langle\nabla V(\omega, u), \pi f_{\omega}\right\rangle>0,
$$

и, таким образом,

для любого $y \in \pi \mathcal{G}(\omega, u), \omega \in \Omega$.

$$
\langle\nabla V(\omega, u), y\rangle>0
$$

Это значит, что $0 \notin \pi \mathcal{G}(\omega, u)$ для $u \in \partial U \cap \operatorname{Ker} l, \omega \in \Omega$ и

$$
\operatorname{deg}\left(\left.\pi \mathcal{G}\right|_{\bar{U}_{\text {Ker } l}}, \bar{U}_{\text {Ker } l}\right)=\operatorname{deg}\left(\nabla V, \bar{U}_{\text {Ker } l}\right) \neq 0,
$$

где $\bar{U}_{\text {Ker } l}=\bar{U} \cap \operatorname{Ker} l$. Таким образом, все условия Теоремы 6 выполнены и задача (1), (2) имеет решение.

\section{4. Примеры.}

4.1. Случайное дифференииальное включение с запаздъванием. Рассмотрим периодическую задачу для случайного дифференциального включения с запаздыванием

$$
\begin{gathered}
x^{\prime}(\omega, t) \in \mathcal{F}(\omega, t, x(t-\tau)) \text { п. в. } t \in[0, T], \\
x(\omega, 0)=x(\omega, T),
\end{gathered}
$$

где мультиотображение $\mathcal{F}: \Omega \times \mathbb{R} \times \mathbb{R}^{n} \rightarrow K v\left(\mathbb{R}^{n}\right)$ удовлетворяет условиям $\left(\mathcal{F}_{t}\right),(\mathcal{F} 1),(\mathcal{F} 2)$.

Теорема 8. Пусть найдутся $\bar{N}>0 u C>0$ mакие, что $\langle x, y\rangle \geqslant C$ для всех $x,\|x\| \geqslant \bar{N}, \omega \in \Omega$ и $y \in \mathcal{F}(\omega, t, x)$. Если мультиотображсение $\mathcal{F}$ ограничено:

$$
\|\mathcal{F}(\omega, t, x)\|:=\max _{y \in \mathcal{F}(\omega, t, x)}\|y\| \leqslant M
$$

$u$

$$
C-\tau M^{2}>0
$$

то задача (5), (6) имеет решение.

Доказательство. Покажем, что $V(\omega, x)=\frac{1}{2}\|x\|^{2}$ является случайной строгой интегральной направляющей функцией для включения (5). Зафиксируем произвольное $\omega \in \Omega$. Тогда для достаточно большого $N>0$ условия

$$
\left\|x^{\prime}(\omega, t)\right\| \leqslant M \text { п. в. } t \in[0, T] \text { и }\|x\|_{2} \geqslant N
$$

будут влечь

$$
\|x(\omega, t)\| \geqslant \bar{N} \text { при всех } t \in[0, T]
$$


для любой абсолютно непрерывной функции

$$
x(\omega, \cdot) \in C_{T} .
$$

Но тогда для такой функции $x(\omega, \cdot)$ мы имеем для произвольного сечения $f_{\omega} \in \mathcal{P}_{\mathcal{F}}(\omega, x)$ :

$$
\begin{aligned}
& \int_{0}^{T}\left\langle\nabla V(\omega, x(s)), f_{\omega}(s)\right\rangle d s= \\
& \quad=\int_{0}^{T}\left\langle x(s), f_{\omega}(s)\right\rangle d s=\int_{0}^{T}\left\langle x(s-\tau), f_{\omega}(s)\right\rangle d s+\int_{0}^{T}\left\langle x(s)-x(s-\tau), f_{\omega}(s)\right\rangle d s= \\
& =\int_{0}^{T}\left\langle x(s-\tau), f_{\omega}(s)\right\rangle d s+\int_{0}^{T}\left\langle\int_{-\tau}^{s} x^{\prime}(s) d s, f_{\omega}(s)\right\rangle d s \geqslant C T-\tau M^{2} T=\left(C-\tau M^{2}\right) T>0 .
\end{aligned}
$$

4.2. Случайное полулинейное функиионально-дифберенциальное включение. Рассмотрим следующую периодическую задачу

$$
\begin{gathered}
x^{\prime}(\omega, t) \in A x(\omega, t)+\mathcal{F}\left(\omega, t, x_{t}\right), \\
x(\omega, 0)=x(\omega, T) .
\end{gathered}
$$

Здесь мультиотображение $\mathcal{F}: \Omega \times \mathbb{R} \times C \rightarrow K v\left(\mathbb{R}^{n}\right)$ удовлетворяет условиям $\left(\mathcal{F}_{t}\right),(\mathcal{F} 1),(\mathcal{F} 2)$, a $A: \mathbb{R}^{n} \rightarrow \mathbb{R}^{n}$ - линейный оператор.

Теорема 9. Пусть квадратичная форма $\langle A x, x\rangle$ удовлетворяет для некоторого $\varepsilon>0$ условию

$$
\langle A x, x\rangle \geqslant \varepsilon\|x\|^{2}
$$

для всех $x \in \mathbb{R}^{n}$. Eсли

$$
\varlimsup_{\|x\|_{2} \rightarrow+\infty} \frac{\left\|\mathcal{P}_{F}(\omega, x)\right\|_{2}}{\|x\|_{2}}<\varepsilon
$$

для всех $\omega \in \Omega$, для любой абсолютно непрерывной функиии $x \in C_{T}$, где $\mathcal{P}_{\mathcal{F}}-$ мультиоператор суперпозиции, порожденный $\mathcal{F}$, a

$$
\left\|\mathcal{P}_{\mathcal{F}}(\omega, x)\right\|_{2}=\sup _{f \in \mathcal{P}_{\mathcal{F}}(\omega, x)}\|f\|_{2},
$$

то задача (7), (8) имеет решение.

Доказательство. Покажем, что $V(\omega, x)=\frac{1}{2}\|x\|^{2}$ является случайной строгой интегральной направляющей функцией для включения (7). Зафиксируем произвольное $\omega \in \Omega$. Тогда для произвольного $f_{\omega} \in \mathcal{P}_{\mathcal{F}}(\omega, x)$ имеем

$$
\begin{array}{r}
\int_{0}^{T}\left\langle\nabla V(\omega, x(s)), A x(s)+f_{\omega}(s)\right\rangle d s=\int_{0}^{T}\langle A x(s), x(s)\rangle d s+\int_{0}^{T}\left\langle x(s), f_{\omega}(s)\right\rangle d s \geqslant \\
\quad \geqslant \varepsilon\|x\|_{2}^{2}-\|x\|_{2}\left\|\mathcal{P}_{\mathcal{F}}(\omega, x)\right\|_{2}>0
\end{array}
$$

для достаточно больших значений $\|x\|_{2}$. 
4.3. Случайное градиентное функиионально-дифференииальное включение. Рассмотрим периодическую задачу вида

$$
\begin{gathered}
x^{\prime}(\omega, t) \in \nabla \mathcal{G}(\omega, x(t))+\mathcal{F}\left(\omega, t, x_{t}\right), \\
x(\omega, 0)=x(\omega, T),
\end{gathered}
$$

где мультиотображение $\mathcal{F}$ удовлетворяет условиям $\left(\mathcal{F}_{t}\right),(\mathcal{F} 1),(\mathcal{F} 2)$, а $\nabla \mathcal{G}$ - градиент случайного потенциала $\mathcal{G}: \Omega \times \mathbb{R}^{n} \rightarrow \mathbb{R}$.

Теорема 10. Пусть выполнены условия:

1. найдутся константы $\varepsilon>0, K>0 u \beta \geqslant 1$ такие, что

$$
\|\nabla \mathcal{G}(\omega, x)\| \geqslant \varepsilon\|x\|^{\beta}-K
$$

для всех $x \in \mathbb{R}^{n}, \omega \in \Omega$;

2. $\varlimsup_{\|x\|_{2} \rightarrow+\infty} \frac{\left\|\mathcal{P}_{\mathcal{F}}(\omega, x)\right\|_{2}}{\|x\|_{2}^{\beta}}<\varepsilon T^{(1-\beta) / 2}$ для всех $\omega \in \Omega$, для любой абсолютно непрерывной функции $x(\omega, \cdot) \in C_{T}$;

3. градиент $\nabla \mathcal{G}$ имеет ненулевой случайный топологический индекс:

$$
\operatorname{Deg}\left(\nabla \mathcal{G}, \partial B_{N}\right) \neq 0
$$

для достаточно больших $N>0$.

Тогда задача (9), (10) имеет решение.

Доказательство. Покажем, что $\mathcal{G}$ является случайной строгой интегральной направляющей функцией для включения (9). Отметим, что вложение $L^{2 \beta} \subset L^{2}$ дает для любой абсолютно непрерывной функции $x(\omega, \cdot) \in C_{T}$ оценку

$$
\|\nabla \mathcal{G}(\omega, x(\omega, \cdot))\|_{2} \geqslant \varepsilon\|x\|_{2 \beta}^{\beta}-K \sqrt{T} \geqslant \varepsilon T^{(1-\beta) / 2}\|x\|_{2}^{\beta}-K \sqrt{T} .
$$

Зафиксируем произвольное $\omega \in \Omega$. Тогда для любого $f \omega \in \mathcal{P}_{\mathcal{F}}(\omega, x)$ имеем

$$
\begin{gathered}
\int_{0}^{T}\left\langle\nabla \mathcal{G}(\omega, x(s)), \nabla \mathcal{G}(\omega, x(s))+f_{\omega}(s)\right\rangle d s \geqslant\|\nabla \mathcal{G}(\omega, x(\omega, \cdot))\|_{2}\left(\|\nabla \mathcal{G}(\omega, x(\omega, \cdot))\|_{2}-\left\|f_{\omega}\right\|_{2}\right) \geqslant \\
\geqslant\|\nabla \mathcal{G}(\omega, x(\omega, \cdot))\|_{2}\left(\|\nabla \mathcal{G}(\omega, x(\omega, \cdot))\|_{2}-\left\|\mathcal{P}_{\mathcal{F}}(\omega, x)\right\|_{2}\right) \geqslant \\
\geqslant\|\nabla \mathcal{G}(\omega, x(\omega, \cdot))\|_{2}\left(\varepsilon T^{(1-\beta) / 2}-\frac{K \sqrt{T}}{\|x\|_{2}^{\beta}}-\frac{\left\|\mathcal{P}_{\mathcal{F}}(\omega, x)\right\|_{2}}{\|x\|_{2}^{\beta}}\right)\|x\|_{2}^{\beta}>0
\end{gathered}
$$

для достаточно больших значений $\|x\|_{2}$.

\section{СПИСОК ЛИТЕРАТУРЫ}

1. Благодатских В. И., Филиппов А. Ф. Дифференциальные включения и оптимальное управление// Тр. Мат. ин-та им. В. А. Стеклова АН СССР. - 1985. - 169. - С. 194-252.

2. Борисович Ю. Г., Гелъман Б. Д., Мышкис А. Д., Обуховский В. В. Введение в теорию многозначных отображений и дифференциальных включений. - М.: Либроком, 2011.

3. Гетманова E. H. О степени совпадения для некоторых классов случайных мультиотображений и линейных фредгольмовых операторов// Материалы Воронежской весенней математической школы «Современные методы теории краевых задач. Понтрягинские чтения-XXX» (3-9 мая 2019 г.). - Воронеж, 2019.

4. Корнев С. В. Негладкие интегральные направляющие функции в задачах о вынужденных колебаниях// Автомат. телемех. - 2015. - 9. - С. 31-43.

5. Корнев C. В. Многолистные направляющие функции в задаче о существовании периодических решений дифференциальных включений с невыпуклой правой частью// Изв. вузов. Мат. - 2016. - 11. C. $14-26$. 
6. Корнев $C$. В. Направляющие функции на заданном множестве в задаче о существовании периодических решений дифференциальных включений с невыпуклой правой частью// Вестн. Воронеж. гос. ун-та. Сер. Физ. Мат. - 2016. - 2. - С. 107-122.

7. Корнев $C$. В. Метод негладких интегральных направляющих функций в задаче о существовании периодических решений включений с каузальными операторами// Вестн. Южно-Уральск. гос. ун-та. Сер. Мат. модел. Программир. - 2016. - 9, № 2. - С. 46-59.

8. Корнев C. В., Обуховский B. В. Об интегральных направляющих функциях для функциональнодифференциальных включений// в кн.: Топологические методы нелинейного анализа. - Воронеж, 2000. - C. $87-107$.

9. Корнев C. В., Обуховский B. В. О некоторых вариантах теории топологической степени для выпуклозначных мультиотображений// в кн.: Труды математического факультета ВГУ. - Воронеж: Воронеж. гос. ун-т, 2004. - С. 56-74.

10. Корнев C. В., Обуховский В. В. О локализации метода направляющих функций в задаче о периодических решениях дифференциальных включений// Изв. вузов. Мат. - 2009. - 5. - С. 23-32.

11. Корнев C. В., Обуховский B. В. Интегральные направляющие функции и периодические решения включений с каузальными операторами// Вестн. Тамбов. ун-та. Сер. Естеств. техн. науки. - 2016. 21, № 1. - C. 55-65.

12. Корнев C. В., Обуховский В. В., Дзекка П. Метод обобщенной интегральной направляющей функции в задаче о существовании периодических решений функционально-дифференциальных включений// Диффер. уравн. - 2016. - 52, № 10. - С. 1335-1344.

13. Красносельский M. А. Оператор сдвига по траекториям дифференциальных уравнений. - М.: Наука, 1966.

14. Красносельский М. А., Забрейко П. П. Геометрические методы нелинейного анализа. - М.: Наука, 1975.

15. Красносельский М. А., Перов А. И. Об одном принципе существования ограниченных, периодических и почти-периодических решений у систем обыкновенных дифференциальных уравнений// Докл. АН CCCP. - 1958. - 123, № 2. - С. 235-238.

16. Красносельский М. А., Перов А. И., Поволоцкий А. И., Забрейко П. П. Векторные поля на плоскости. - М.: Физматгиз, 1963.

17. Толстоногов А. А. Дифференциальные включения в банаховом пространстве. - Новосибирск: Наука, 1986.

18. Andres J., Gorniewicz L. Random topological degree and random differential inclusions// Topol. Meth. Nonlin. Anal. — 2012. - 40. — P. 337-358.

19. Arutyunov A. V., Obukhovskii V. Convex and Set-Valued Analysis. Selected Topics. - Berlin-Boston: de Gruyter, 2017.

20. Deimling K. Multivalued Differential Equations. — Berlin-New York: de Gruyter, 1992.

21. Fonda A. Guiding functions and periodic solutions to functional differential equations// Proc. Am. Math. Soc. - 1987. - 99, № 1. - P. 79-85.

22. Gliklikh Yu., Kornev S., Obukhovskii V. Guiding potentials and periodic solutions of differential equations on manifolds// Glob. Stochast. Anal. — 2019. - 6, № 1. - P. 1-7.

23. Górniewicz L. Topological Fixed Point Theory of Multivalued Mappings. — Berlin: Springer-Verlag, 2006.

24. Kamenskii M., Obukhovskii V., Zecca P. Condensing Multivalued Maps and Semilinear Differential Inclusions in Banach Spaces. - Berlin-New York: de Gruyter, 2001.

25. Kisielewicz M. Differential Inclusions and Optimal Control. - Dordrecht: PWN, 1991.

26. Kornev S., Obukhovskii $V$. On some developments of the method of integral guiding functions// Funct. Differ. Equ. - 2005. - 12, № 3-4. - P. 303-310.

27. Kornev S. V., Liou Y. C., Loi N. V., Obukhovskii V. V. On periodic solutions of random differential inclusions// Appl. Anal. Optim. - 2017. — 1, № 2. - P. 245-258.

28. Kornev S. V., Loi N. V., Obukhovskii V. V., Wen C. F. Random nonsmooth integral guiding functions and asymptotic behavior of trajectories for random differential inclusions// J. Nonlin. Convex Anal. — 2018. - 19, № 3. - P. 493-500.

29. Kornev S., Obukhovskii V., Zecca P. Guiding functions and periodic solutions for inclusions with causal multioperators// Appl. Anal. - 2017. - 96, № 3. - P. 418-428.

30. Kornev S., Obukhovskii V., Zecca P. On multivalent guiding functions method in the periodic problem for random differential equations// J. Dynam. Differ. Equ. — 2019. — 31, № 2. — P. 1017-1028. 
31. Mawhin J. L. Topological Degree Methods in Nonlinear Boundary Value Problems. — Providence, Rhode Island: Am. Math. Soc., 1979.

32. Mawhin J., Thompson H. B. Periodic or bounded solutions of Carathéodory systems of ordinary differential equations// J. Dynam. Differ. Equ. — 2003. — 15, № 2-3. — P. 327-334.

33. Mawhin J., Ward J. R. Jr. Guiding-like functions for periodic or bounded solutions of ordinary differential equations// Discr. Contin. Dynam. Syst. - 2002. - 8, № 1. - P. 39-54.

34. Obukhovskii V., Zecca P., Loi N. V., Kornev S. Method of Guiding Functions in Problems of Nonlinear Analysis. - Berlin: Springer-Verlag, 2013.

35. Pruszko T. A coincidence degree for $L$-compact convex-valued mappings and its application to the Picard problem for orientor fields// Bull. Acad. Pol. Sci. Sér. Math. — 1979. — 27, № 11-12. — P. 895-902.

36. Pruszko T. Topological degree methods in multi-valued boundary value problems// Nonlin. Anal. Theory Meth. Appl. - 1981. — 5, № 9. - P. 959-970.

37. Rachinskii D. I. Multivalent guiding functions in forced oscillation problems// Nonlin. Anal. Theory Meth. Appl. - 1996. - 26. - P. 631-639.

38. Tarafdar E., Teo S. K. On the existence of solutions of the equation $L x \in N x$ and a coincidence degree theory// J. Austr. Math. Soc. - 1979. - A. 28, № 2. - P. 139-173.

39. Tarafdar E., Watson P., Yuan X. Z. Random coincidence degree theory with applications to random differential inclusions// Comment. Math. Univ. Carol. — 1996. — P. 725-748.

Гетманова Екатерина Николаевна

Воронежский государственный педагогический университет

E-mail: ekaterina_getmanova@bk.ru

Корнев Сергей Викторович

Воронежский государственный педагогический университет

E-mail: kornev_vrn@rambler.ru 